\title{
Quasistatic thermal and nonlinear processes of photoconver- sion of high-density optical radiation by multilayer structures
}

\author{
Arkadiy Blank ${ }^{1,2, \star}$, Anton Razuvaev ${ }^{1}$, Natalia Suhareva ${ }^{2}$, and Vjatcheslav Tugaenko ${ }^{1}$ \\ ${ }^{1}$ S.P. Korolev Rocket and Space Corporation "Energia" (Russian Federation) \\ ${ }^{2}$ M.V. Lomonosov Moscow State University (Russian Federation)
}

\begin{abstract}
The results of the systematic experimental analysis of the thermal nonlinear electro-optic properties of photoelectric converters with silicon vertical cells in comparison with solar elements and elements on the basis of $\mathrm{In} / \mathrm{Ga} / \mathrm{As}$ are presented. The parameters of the linear and quadratic approximations for the investigated dependences are determined, that allows constructing a scalable analytic model of the converter with a given type of the working elements switching.
\end{abstract}

Perspective technologies of the directed delivery of powerful spatially concentrated continuous energy flows are associated with high-density photoelectric converters, ensuring much higher efficiency of photoelectric transformation than that of the Shock-Quiester threshold for single-layer systems. A typical example of such converters designed for flow densities over $10 \mathrm{~W} / \mathrm{cm}^{2}$ is a layered silicon structure offered by MH GoPower [1,2]. Real operating conditions for such photo converters revealed a number of significant features and limitations, associated essentially with the thermal- and photoself-action of the converted flow at high radiation concentrations and insufficient heat removal from the site. First of all, this is the thermal drift of the characteristics, the nonlinearity of the response to the changes in the radiation flow density, and the thermodynamic instability of the system.
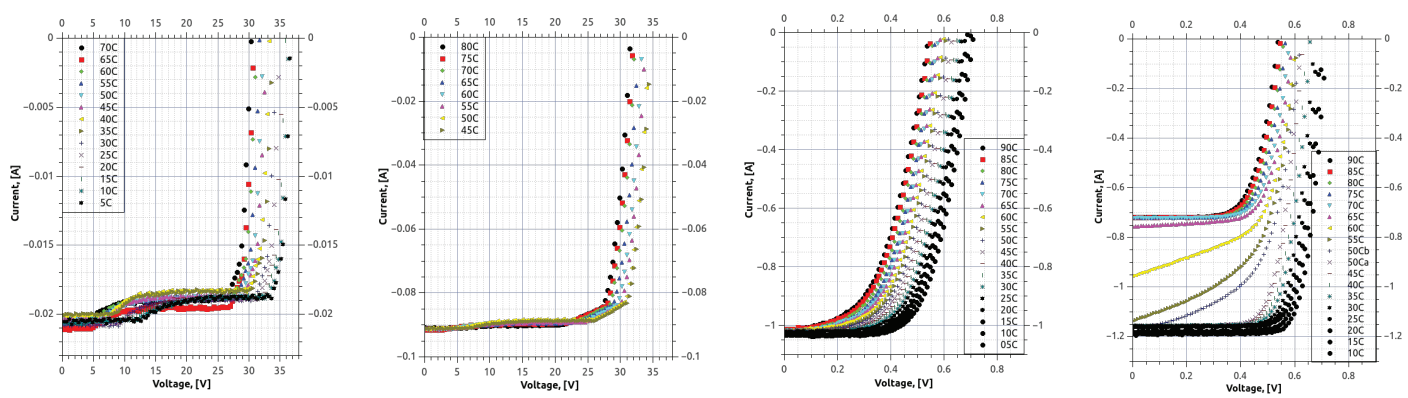

Figure 1. Example of the i-v characteristic for Si vertical cells (left and left-middle for various beam power), solar cell (right-middle) and InGaAs cell (right)

\footnotetext{
^e-mail: blankarkadiy@physics.msu.ru
} 
In the experiments we used the sources of single-mode and multimode continuous radiation having power up to $120 \mathrm{~W}$ and operating at wavelengths of $808 \mathrm{~nm}$ and $1082 \mathrm{~nm}$. The choice of specific wavelengths reconciles the energy band structure of the silicon converter. The collimated beam covered the sample almost uniformly, the temperature stabilization of the sample surface was provided by the Peltier element, the spatial temperature profile of the site and the laser beam profile was fixated in the wavelength range from $12 \mu \mathrm{m}$ to the visible range.

The control of the photoelectric conversion process is based on the analysis of the set of the $\mathrm{i}-\mathrm{v}$ characteristics control values. They are considered to represent the sequence of the change in the photoconductor operating mode during the applied voltage change (Fig. 1). To measure the i-v characteristics a four-point scheme for different temperatures of the PVC surface, the photocurrent density, and the wavelength was used. Significant temperature drops in the range from $-80^{\circ} \mathrm{C}$ up to $+130^{\circ} \mathrm{C}$, arising during the operation of space-based photo convertors, are accompanied by the drift of the boundaries of the spectral conversion band and the change in the carriers concentration. The reversed directivity of the characteristics is related to the position of the generation frequencies in the photo conversion band, since $\lambda=1062 \mathrm{~nm}$ lies on the red band boundary. Modules of the temperature coefficients dependence at the wavelength inside the range near to its edge $\left(+0,0007^{\circ} \mathrm{C}-1\right.$ and $-0,0009^{\circ} \mathrm{C}-1$ ) are close in magnitude within the measurement inaccuracy.

The maximum power produced by the FEP at the energy densities of the laser beam used in the temperature range from $0^{\circ} \mathrm{C}$ to $+90^{\circ} \mathrm{C}$ can be calculated from the semi-empirical equation of a quasi-stationary process using a linear approximation of the temperature dependence and quadratic dependence of the flow density of photo radiation:

$$
W_{P V C}=\alpha_{1}(T) W_{L B}+\alpha_{2}(T) W_{L B}^{2}
$$

here $W_{L B}$ - is laser beam power, $W_{P V C}-$ is power generated by the solar cells, parameters $\alpha_{1}, \alpha_{2}$ are defined as follows:

$$
\alpha_{1}(T)=0.14-3.3 * 10^{-4} * T, \alpha_{2}(T)=-7.7 * 10^{-4}+2.5 * 10^{-5} * T .
$$

Note that both parametric dependencies presented are sign-alternating. If one needs to estimate the partial contributions of the of multi-section FEP matrices having known temperature distribution and radiation flow density over the surface their using allows to solve a wide range of technological problems of selecting the optimal elements commutation for a given type of energy-carrying beams.

The work was supported by Russian Foundation for Basic Research. RFBR Project A-15-0807484 .

\section{References}

[1] Perales, Mico, et al. "Characterization of high performance silicon-based VMJ PV cells for laser power transmission applications.", Proc. of SPIE 9733, (2016)

[2] Perales, Mico, et al. "Design and characterization of a novel power over fiber system integrating a high power diode laser.", Proc. of SPIE 10086, (2017) 